

No. 82

February 1986

An occasional series reporting on U.S. and international GARP scientific, technical, and planning activities, developments, and programs, presented as a public service to the meteorological community by the American Meteorological Society through arrangements with the U.S. Committee on the Global Atmospheric Research Program of the National Academy of SciencesNational Research Council. Opinions expressed in "GARP Topics" do not necessarily reflect the point of view of the U.S. Committee.

\title{
The Observational Error of Automated Wind Reports from Aircraft
}

\author{
Lauren L. Morone ${ }^{1}$
}

\begin{abstract}
Data collected from aircraft equipped with AIDS (Aircraft Integrated Data System) instrumentation during the Global Weather Experiment year of 1979 are used to estimate the observational error of winds at flight level from this and other aircraft automated windreporting systems. Structure functions are computed from reports that are paired using specific criteria. The value of this function extrapolated to zero separation distance is an estimate of twice the random measurement-error variance of the AIDS-measured winds. Component-wind errors computed in this way range from 2.1 to 3.1 $\mathrm{m} \cdot \mathrm{s}^{-1}$ for the two months of data examined, January and August 1979. Observational error, specified in optimum-interpolation analyses to allow the analysis to distinguish among observations of differing quality, is composed of both measurement error and the error of unrepresentativeness. The latter type of error is a function of the resolvable scale of the analysis-prediction system. The structure function, which measures the variability of a field as a function of separation distance, includes both of these types of error. If the resolvable scale of an analysis procedure is known, an estimate of the observational error can be computed from the structure function at that particular distance. An observational error of $5.3 \mathrm{~m} \cdot \mathrm{s}^{-1}$ was computed for the $u$ and $v$ wind components for a sample resolvable scale of 300 $\mathrm{km}$. The errors computed from the structure functions are compared to colocation statistics from radiosondes. The errors associated with automated wind reports are found to compare favorably with those estimated for radiosonde winds at that level.
\end{abstract}

\footnotetext{
' Development Division, National Meteorological Center, NWS/ NOAA, Washington, DC 20233
}

\section{Introduction}

This paper is a report on a study to determine the observational-error variance of winds measured by inertial navigation systems (INS) on wide-bodied jet aircraft. There are three types of this kind of data, which differ in the method of transmission. All of them come from the same automated package that records wind speed, direction, temperature, pressure, location, and many other parameters from the INS of the aircraft and other instrumentation on board (Julian and Steinberg, 1975). AIDS stands for the Aircraft Integrated Data System, in which the data are recorded on tape on board the aircraft and removed after the flight. The delay involved is too great to allow its operational use. ASDAR (Aircraft to Satellite Data Relay) transmits the observations from the aircraft during the flight to a geostationary satellite which relays them to a ground station for entry into the Global Telecommunications System for use by numerical-weather-prediction centers. ACARS (ARINC [Aeronautical Radio, Incorporated] Communications Addressing and Reporting System) reports are transmitted directly to ground stations. AIDS data alone are used in this study but all of the results can be applied to ASDAR and ACARS data as well. The AIDS data base was taken from the Global Weather Experiment year of 1979 .

Optimum interpolation (OI) is a statistical objective-analysis procedure in use at the National Meteorological Center 
and at many other prediction centers around the world (Bergman, 1979). INS winds reported automatically are used in these analyses. The OI procedure is able to distinguish among observations of differing types and quality by means of a prespecified observational-error standard deviation assigned to each type. It is important to know this quantity to make the best use of a report from any type of instrument.

Observational error is defined as the difference between an observed value and "truth." In the context of objective analysis, truth is defined as an accurate description of only the scales of motion resolvable by the analysis (Lorenc, 1984) and is, therefore, a function of analysis. Observational error can be considered to arise from two separate sources as discussed by Julian (1983). Measurement error refers to the difference between two measurements of the same quantity by two different instruments. The other source of error is due to the inability of a point measurement to provide a value that is representative of the grid and time scale appropriate for the particular analysis, i.e., "truth." Julian (1983) calls this the error of unrepresentativeness. This study provides estimates for both of these sources of error for INS-measured winds reported by AIDS, ASDAR, and ACARS.

\section{The method}

An investigation of the statistical structure of the INS wind data was chosen as the best means of providing information on the observational error of the data. This method takes advantage of the frequent reporting interval of INS winds. It uses pairs of wind observations from different flights separated by relatively short distances and times. Many researchers have used statistical analysis of meteorological observations to reveal details of the scales of atmospheric variability (Barnes and Lilly, 1975; Hillger and Vonder Haar, 1979; Maddox and Vonder Harr, 1979; Fuelberg and Meyer, 1984). Specifically, they have calculated structure and correlation functions from the observations. By-products of these investigations have been estimates of the measurement error of the particular type of instrument involved.

The structure function appropriate for meteorological variables at the points defined by position vectors $s_{1}$ and $s_{2}$ is defined by Gandin (1963) as

$$
b_{f}\left(s_{1}, s_{2}\right)=\overline{\left[f^{\prime}\left(s_{1}\right)-f^{\prime}\left(s_{2}\right)\right]^{2}},
$$

where

$$
f^{\prime}\left(s_{i}\right)=f\left(s_{i}\right)-\bar{f}\left(s_{i}\right)
$$

and $\bar{f}(s)$ is the climatological mean of the variable. The data for this study are paired aircraft wind observations collected over a period of one month and located all over the Northern Hemisphere. Since the paired observations seldom if ever reoccur at the same geographical location, it is impossible to calculate a local climatological mean for a particular $\left(s_{1}, s_{2}\right)$. Therefore, in order to use this type of data set, the definition of the structure function has been modified as in Hillger and Vonder Haar (1979), Maddox and Vonder Haar (1979), and Fuelberg and Meyer (1984) to allow the mean $\bar{f}(s)$ to be defined as a spatial mean over a specified distance interval.
Two assumptions are customary in applying structurefunction analysis to a meteorological data set of this type. The first is of statistical homogeneity. Under this assumption, the structure function is independent of the location of the pair of observations over the geographical area of study. The second assumption is that of isotropy. An isotropic structure function is independent of the orientation of the two observations that make up the pair.

Combining these assumptions results in a structure function that depends only on the separation distance between the paired reports. Equation (1) then becomes

$$
b_{f}(s)=\overline{\left[f\left(s_{i}\right)-f\left(s_{j}\right)\right]^{2}},
$$

where $s$ represents a specified distance equal to $\left|s_{i}-s_{j}\right|$ and the mean is computed over such pairs that have separation distance $s$.

In this study, as in the studies previously referenced, $s$ refers not to a specific distance $\left|s_{i}-s_{j}\right|$, but to a distance interval. Pairs were constructed with separations of up to $500 \mathrm{~km}$ and structure functions were computed by averaging over all the pairs whose distance separations fell within specified 25$\mathrm{km}$ intervals $(0-25 \mathrm{~km}, 26-50 \mathrm{~km}$, etc.). Note also that the observed variable $f$ appears in (3) and not $f^{\prime}$. The horizontal means cancel out of the expression due to the additional assumption that the $f$ field is spatially homogeneous. This assumption, also made by Hillger and Vonder Haar (1979), Maddox and Vonder Haar (1979), and Fuelberg and Meyer (1984), will introduce some error into the structure computations that follow. However, an attempt to reduce this source of error has been made by placing certain restrictions on the pairings of the aircraft wind observations during the selection procedure. The data have been stratified by season and pairs are selected away from regions of strong horizontal shear such as jet streams. The selection process is described in detail in Section 3.

The observed value $f$ can be expressed as the sum of its true value $f_{T}$ and the deviation from its true value $\delta$. Assuming the error $\delta$ is random, Gandin (1963) derives the following equation from (3) for the structure function, relying also on the homogeneity and isotropic assumptions:

$$
b_{f}(s)=b_{f_{r}}(s)+2 \sigma_{f}^{2} .
$$

The mean-square error $\overline{\delta^{2}}$ is represented as $\sigma_{f}{ }^{2}$. At zero separation, the structure function of the error-free data, $b_{f_{T}}(0)$, is zero, so (4) becomes

$$
b_{f}(0)=2 \sigma_{f}^{2} .
$$

This then is the method by which an estimate of the measurement error is obtained. Structure functions are calculated by the method presented above, plotted as a function of distance, and the resulting curves are extrapolated to zero separation. It is important to remember that the effectiveness of this method relies heavily on how well the data set obeys the given assumptions.

Correlation functions can also be computed from this data set. Using the assumptions necessary to derive (3), the equation for the structure function, the correlation function equation is

$$
m_{f}(s)=\frac{\overline{\left[f^{\prime}\left(s_{i}\right) f^{\prime}\left(s_{j}\right)\right]}}{\overline{f^{\prime}\left(s_{i}\right)^{2}}}
$$


It is helpful to recall that $s$ denotes an interval of separation, the bar symbolizes a mean over all the pairs within that interval, and $f^{\prime}(s)$ is the deviation of the observed value from the spatial mean $[\bar{f}(s)]$ computed within that same separation interval (Fuelberg and Meyer, 1984).

Correlation functions are similar to structure functions in the information they provide on the variability of the atmosphere as measured by a particular instrument. Since it is a normalized quantity, correlations from one type of instrument can be easily compared to instruments of other types and they are included in this paper for that purpose.

Random errors affect correlation function calculations such that the correlation is not exactly 1.0 at zero separation. This is because the observed variance (the denominator in [6]) at zero separation $\left(\operatorname{VAR}_{f}[0]\right)$ is larger than the true variance by an amount equal to the mean-square error attributable to the instrument, i.e.,

$$
\operatorname{VAR}_{f}(0)=\operatorname{VAR}_{T}(0)+\sigma_{f}^{2} .
$$

The technique described by Hillger and Vonder Haar (1979), which corrects for that error, results in an expression for the correlation function,

$$
m_{f}(s)=\frac{m_{f_{T}}(s)}{1+\left[\sigma_{f}^{2} / \operatorname{VAR}_{T}(0)\right]}
$$

Since the true correlation at zero separation is 1.0 and the value of the observed correlation at that separation can be extrapolated from the values computed using (6), the value of $\operatorname{VAR}_{T}(0)$ can be derived by setting $s$ to zero separation in (8) and using the mean-square error $\sigma_{f}^{2}$ computed from (5). $\operatorname{VAR}_{T}(0)$ is then used with $\sigma_{f}^{2}$ to increase the observed correlations from (6) as described by (8). The correlation-function values presented in this paper were computed and corrected using this procedure.

\section{Data collection}

Data for two seasons were analyzed separately in this study. The winter-season data set consisted of 29 days of INSmeasured, AIDS-recorded (INS-AIDS) wind observations from the month of January 1979. The summer season was represented by 27 days of INS-AIDS observations from August 1979. For each season only Northern Hemisphere reports were considered. For each available day of the two data sets, at 00 GMT and 12 GMT, each INS-AIDS report was identified within the data file and an attempt was made to pair it with another report from a different flight. Two INSAIDS reports constituted a pair if:

1) each report originated from a different flight,

2) the two reports were within $500 \mathrm{~km}$ and three hours of each other,

3) they had a vertical separation of no more than 100 meters,

4) they were both above 9000 meters, and

5) the wind speed of both reports was less than or equal to $20 \mathrm{~m} \cdot \mathrm{s}^{-1}$.

Condition 4 had the effect of restricting all the pairs to the aircraft's cruising altitude rather than the ascending or de-
TABLE 1. The total number of pairs and their distribution per interval for January and August 1979.

\begin{tabular}{ccc}
\hline $\begin{array}{c}\text { Separation Interval } \\
(\mathrm{km})\end{array}$ & January & August \\
\hline $0-25$ & 122 & 286 \\
$25-50$ & 132 & 399 \\
$50-75$ & 125 & 392 \\
$75-100$ & 138 & 396 \\
$100-125$ & 139 & 443 \\
$125-150$ & 202 & 472 \\
$150-175$ & 199 & 570 \\
$175-200$ & 227 & 678 \\
$200-225$ & 324 & 911 \\
$225-250$ & 333 & 987 \\
$250-275$ & 280 & 901 \\
$275-300$ & 292 & 931 \\
$300-325$ & 310 & 929 \\
$325-350$ & 330 & 897 \\
$350-375$ & 340 & 1029 \\
$375-400$ & 290 & 1008 \\
$400-425$ & 336 & 1037 \\
$425-450$ & 322 & 1181 \\
$450-475$ & 307 & 1123 \\
$475-500$ & 329 & 1012 \\
& & \\
Total & 5077 & 15582 \\
\hline
\end{tabular}

scending leg of the trip. Thus, all pairs can be assumed to be at approximately the same level. In practice, the assumption was a good one. Most of the pairs were located at altitudes between 9500 and 10500 meters. Conditions 3 and 5 were provided in an effort to make the data set more homogeneous and isotropic. Condition 3 reduces the effect of vertical shear and 5 acts to insure that chosen pairs are away from regions of strong jets (horizontal shear). The pairs that meet these criteria are sorted according to their separation distance into 20 intervals of $25 \mathrm{~km}$ each. The total number of pairs and their distribution per interval is shown in Table 1 for both months.

In this study, pairs of observations are grouped only according to their distance separation. Their separation in time is ignored. The validity of this practice is proven by examining the structure functions for specific time separations for any evidence of significant and consistent behavior with respect to time. This was done for both wind components for each month; Fig. 1 presents results for the $u$ component from the January data set. In Fig. 1a six structure functions are plotted as a function of distance, each one computed from pairs of observations whose time separations are within a specific half-hour time interval. In each structure function a significant variation with distance is obvious but a trend among the structure functions themselves with respect to time is not. Fig. lb shows the lack of variation with time more clearly. In it structure functions that have been computed over $100-\mathrm{km}$ distance intervals have been plotted as a function of halfhour time separations. Clearly the decision to compute the structure function solely as a function of distance separation is justified.

\section{Structure and correlation functions}

The structure functions for both wind components computed from the January 1979 data can be found in Fig. 2. As de- 

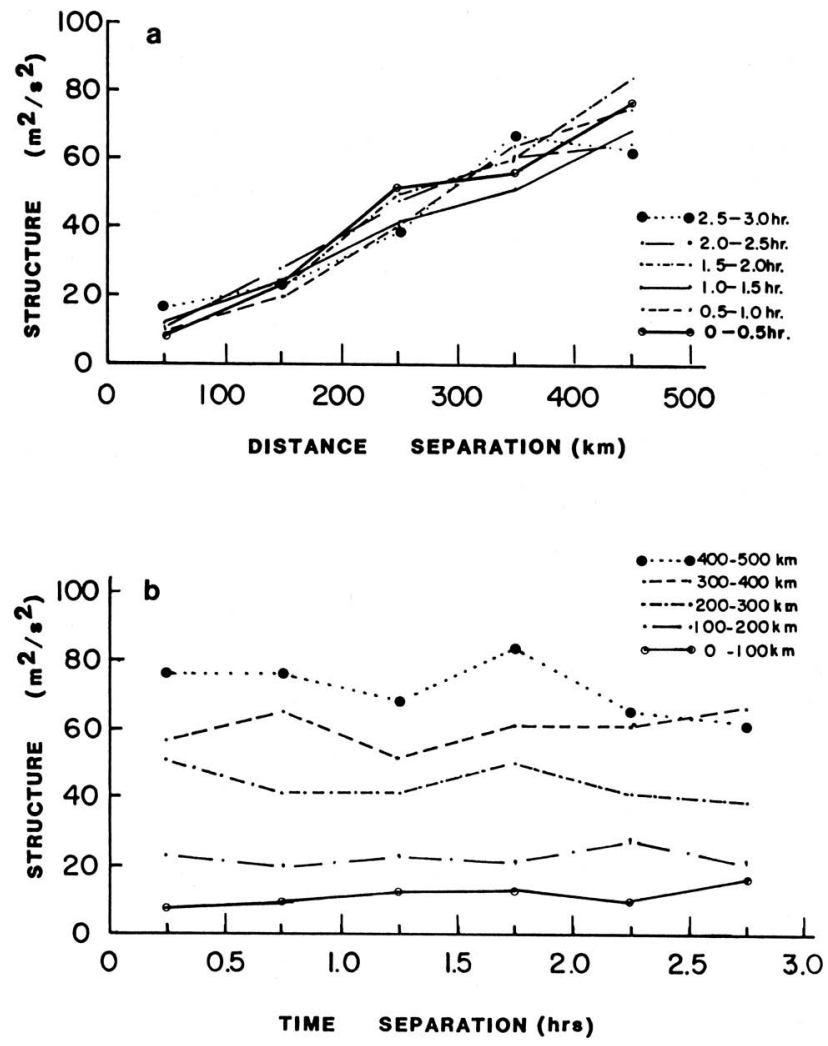

FIG. 1. Structure function of $u$ component of January 1979 data base (a) as a function of distance for each of six half-hour time intervals and (b) as a function of time for each of five $100-\mathrm{km}$ distance intervals.

scribed in Section 2, structure functions were computed for separation distance intervals of $25 \mathrm{~km}$ and are plotted in the figures at the midpoints of the intervals. Also, most of these winds are located between 9500 and 10500 meters, or about $250 \mathrm{mb}$. Fig. 2 shows similar structure for both the $u$ and $v$ wind components. This similarity is also seen between the $u$ and $v$ components in Fig. 3, which shows the structure functions computed from the August 1979 data.

The slope of a structure function computed using the isotropy assumption can be considered to be a measure of the mean nondirectional gradient of a field (Hillger and Vonder Haar, 1979). Since each of these data sets was constructed using measurements collected over an entire month for an entire hemisphere, it is difficult to use this characteristic of the structure function to draw any but the broadest conclusions about the gradients of the $u$ and $v$ fields during these two months. A comparison of Fig. 2 with Fig. 3 shows the August data to have a slightly steeper slope than the January data at smaller separation intervals. At the largest separation intervals, the January data have a slightly steeper slope. Thus, at large scales the winter data show stronger gradients and at shorter scales the summer data show stronger gradients. Overall, however, the structure functions from the two months are quite similar. Most likely, they owe that similarity to the restriction of the measured wind speed to 20 $\mathrm{m} \cdot \mathrm{s}^{-1}$ or less.

Figures 4 and 5 present structure functions as a function of latitude. Figure 4 shows structure functions of the $u$ compo-

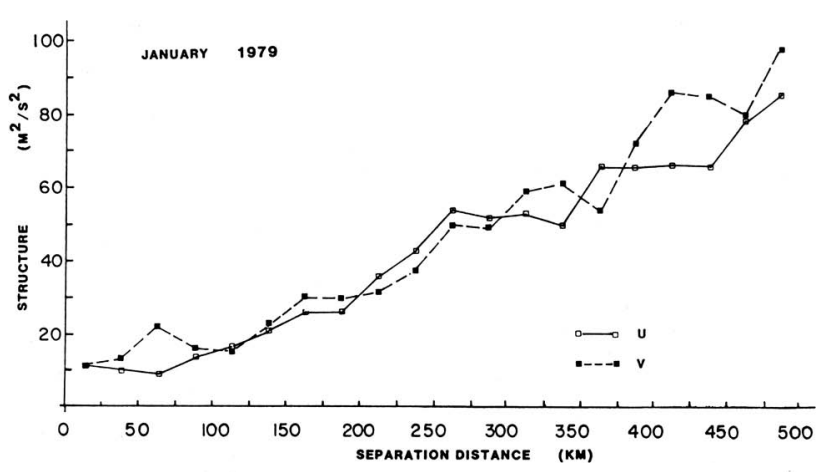

FIG. 2. Structure functions for $u$ and $v$ component of the wind from January 1979 AIDS reports.

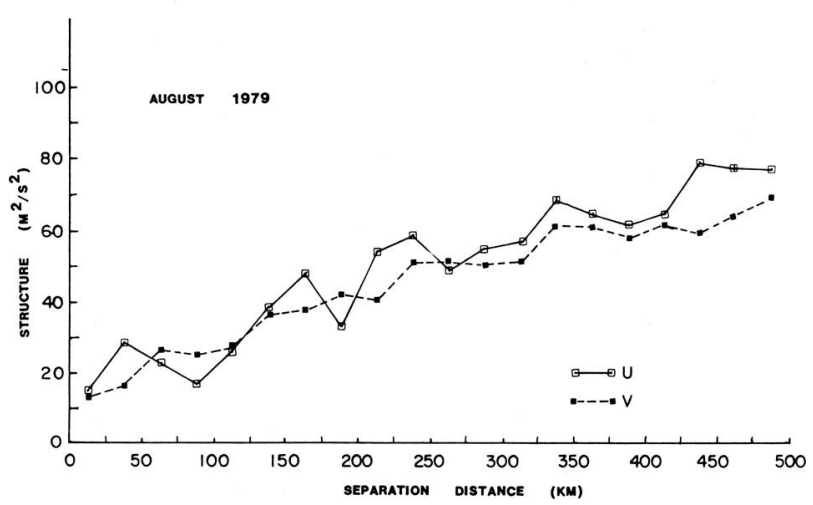

FIG. 3. As in Fig. 2 but for August 1979.

nent of the wind computed from the August data for three latitude bands: $0-30^{\circ} \mathrm{N}, 30-60^{\circ} \mathrm{N}$, and $60-90^{\circ} \mathrm{N}$. Figure 5 is the same but for the $v$ component. The same plots made from the January data are not shown but are quite similar. Most evident from this distribution is the lack of slope associated with the structure function for the $0-30^{\circ} \mathrm{N}$ band. This illustrates the general absence of strong gradients of wind in the tropics. The middle and northernmost latitude bands both show structure functions different from that of the $0-30^{\circ} \mathrm{N}$ band, most strikingly at the larger separation intervals. The structure functions from these two latitude bands are quite similar and represent the stronger gradients of wind found in those regions. Since more than $80 \%$ of the pairs collected in each month were from those two latitude bands, that structure dominates in the structure functions constructed from all the Northern Hemisphere data (Figs. 2 and 3).

As previously emphasized, the assumptions of homogeneity and isotropy are essential to deriving the definition of the structure function used in this study. Figures 4 and 5 provide a rough test of the homogeneity assumption. It appears to be valid north of $30^{\circ} \mathrm{N}$. The isotropy assumption can be studied with the use of Figs. 6 and 7. To construct them, the structure values, already computed as a function of distance interval, are also separated with respect to the angle of the separation vector between each pair. To insure that a sufficient number of pairs were available to compute these structure values, the distance interval was increased to $50 \mathrm{~km}$. The angle of separation was computed with respect to the first re- 


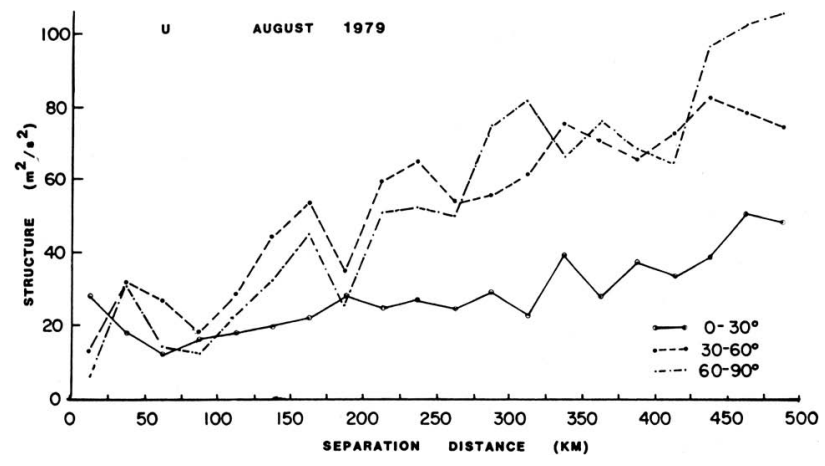

FIG. 4. Structure function of the August $1979 u$ component as a function of three latitude bands: $0-30^{\circ} \mathrm{N}, 30-60^{\circ} \mathrm{N}$, and $60-90^{\circ} \mathrm{N}$.

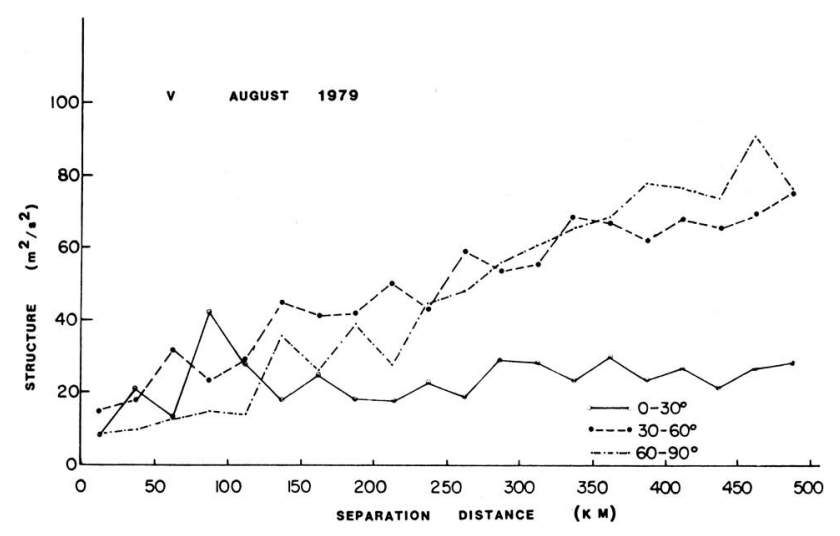

FIG. 5. As in Fig. 4 but for the $v$ component.

port of each pair. In Fig. 6, the structure function for the $u$ wind component is plotted as a function of the angle of separation for two specific distance intervals: $0-50 \mathrm{~km}$, the closest interval, and $450-500 \mathrm{~km}$, the farthest interval. Both the January and the August data are plotted. The structure functions have been averaged over 30-degree intervals and are plotted at the midpoints of those intervals. Figure 7 is the same but for the $v$ component. The most notable characteristic of these plots is the regular variation of both the $u$ and $v$ structure with separation angle at the large interval. This indicates that the fields are highly anisotropic at this distance. The maximum and minimum values of the structure for the $u$ and $v$ components are roughly 90 degrees out of phase. The maximum values on this chart correspond to the orientation in which the field shows the greatest variance over $500 \mathrm{~km}$. For the $u$ component this direction is roughly north and south. For the $v$ component, the direction is east and west. The latter agrees with a plot of the anisotropic character of the $v$ component presented by Maddox and Vonder Haar (1979).

In contrast, at the closest interval the structure functions are relatively constant with angle with perhaps the exception of the $u$-component structure from the August data. Thus these structure functions can be considered relatively isotropic at the closest interval. To determine the distance at which the structure functions can no longer be considered isotropic, plots of the structure functions as a function of the angle of separation were examined for all the distance intervals. The

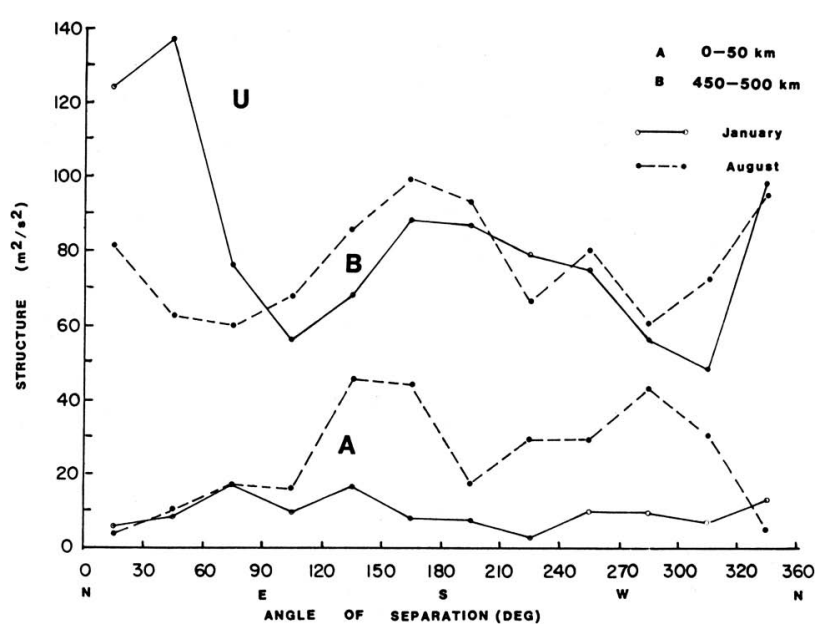

FIG. 6. Structure function of the $u$ component from both the January and August 1979 data as a function of angle of separation for two distance intervals: $0-50 \mathrm{~km}$ and $450-500 \mathrm{~km}$.

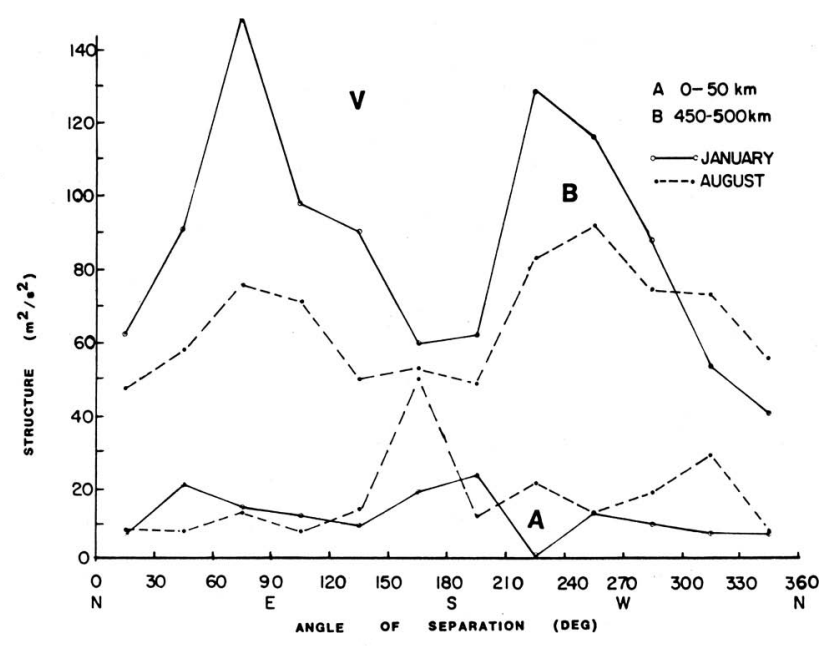

FIG. 7. As in Fig. 6 but for the $v$ component.

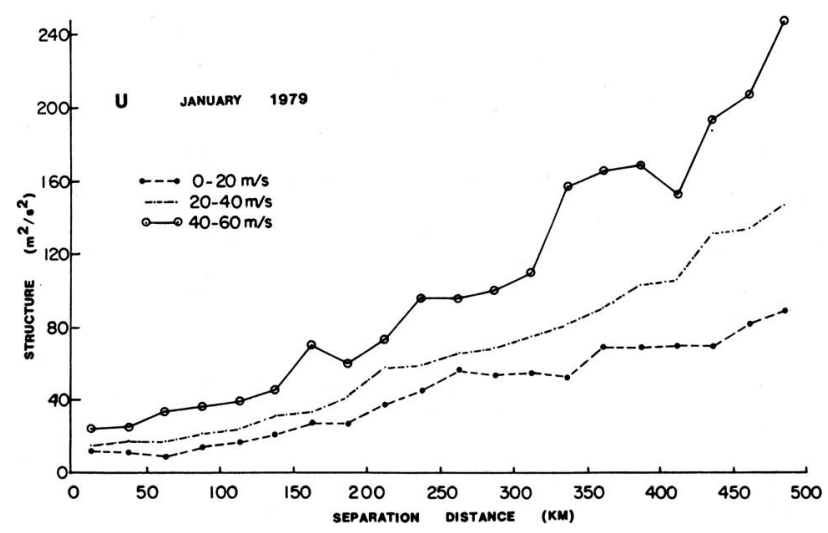

FIG. 8. Structure function of the $u$ component from the January data for three wind-speed intervals: $0-20 \mathrm{~m} \cdot \mathrm{s}^{-1}, 20-40 \mathrm{~m} \cdot \mathrm{s}^{-1}$, and $40-60 \mathrm{~m} \cdot \mathrm{s}^{-1}$. 




FIG. 9. Correlation functions of the $u$ and $v$ components from the January 1979 data.

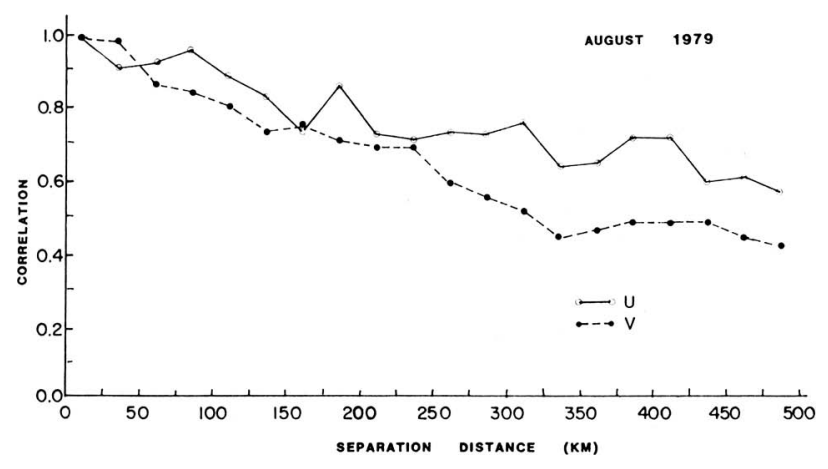

FIG. 10. As in Fig. 9 but from the August 1979 data.

point at which the plots lose their relative constancy and take on the character of the plot of the largest distance interval is approximately $200 \mathrm{~km}$.

One of the criteria used to pair the INS-AIDS wind reports restricted the wind speed of each report to $20 \mathrm{~m} \cdot \mathrm{s}^{-1}$ or less. This was done to reduce the occurrence of paired reports in the vicinity of jet streams, which are regions of strong inhomogeneity and anisotropy. To examine the effect of the speed of the wind on the structure functions, they were computed for two additional intervals of wind speed: $20-40 \mathrm{~m} \cdot \mathrm{s}^{-1}$ and $40-60 \mathrm{~m} \cdot \mathrm{s}^{-1}$. All other pairing criteria remained the same. Fig. 8 shows three structure functions that were computed for the $u$ component from the January data set. The $0-20 \mathrm{~m} \cdot \mathrm{s}^{-1}$ structure function is the same as the $u$ structure function shown in Fig. 2. Note that the ordinate scale has been doubled. As the wind speed increases so does the value of the structure function at all distance intervals. The intercept also increases, which implies an increase in the random measurement error associated with the instrument. However, there is no evidence to suggest that the instrument error associated with INS-derived winds is a function of wind speed (D. Lenschow, personal communication). Therefore, the results shown in Fig. 8 are an illustration of how this technique of computing structure and estimating the measurement error can break down when the assumptions of homogeneity and isotropy no longer apply. The increase of anisotropy was verified by plotting the structure function for wind speeds of $40-60 \mathrm{~m} \cdot \mathrm{s}^{-1}$ as a function of the angle of separation between each pair of reports. These plots showed that the structure values varied with angle even at the smallest
TABLE 2. INS-AIDS error standard-deviation values for the $u$, $v$, and vector wind for January and August 1979 as computed from intercepts of structure functions. Also, radiosonde-speed errors from Fuelberg and Meyer (1984) computed similarly.

\begin{tabular}{ccccc}
\hline \hline Parameter & $\begin{array}{c}u \\
\left(\mathrm{~m} \cdot \mathrm{s}^{-1}\right)\end{array}$ & $\begin{array}{c}v \\
\left(\mathrm{~m} \cdot \mathrm{s}^{-1}\right)\end{array}$ & $\begin{array}{c}V \\
\left(\mathrm{~m} \cdot \mathrm{s}^{-1}\right)\end{array}$ & $\begin{array}{c}\text { Speed } \\
\left(\mathrm{m} \cdot \mathrm{s}^{-1}\right)\end{array}$ \\
\hline \multicolumn{7}{c}{ INS-AIDS winds } \\
January & 2.1 & 2.6 & 3.3 & - \\
August & 3.1 & $\begin{array}{c}\text { 3.1 } \\
\text { Radiosonde winds }\end{array}$ & 4.4 & - \\
$300 \mathrm{mb}$ & - & - & - & 5.4 \\
$200 \mathrm{mb}$ & - & - & - & 6.2 \\
\hline
\end{tabular}

separation interval. Similar results were found for the other data sets as well.

The correlation functions for the $u$ and $v$ wind components from the January and August data are shown in Figs. 9 and 10 respectively. The plotted functions have been corrected to account for the random measurement error as described in Section 2. The correlations computed from the January data in Fig. 9 are quite similar for the two wind components. The similarity between the correlations computed from the $\mathrm{Au}-$ gust data is not as great (Fig. 10). The correlation of the August $v$ component drops more rapidly than that of the $u$ component, especially at separation distances of more than 250 $\mathrm{km}$. A comparison of the January and August correlation functions reveals the same characteristic differences between the two months as when the structure functions for the two months were compared. The slope of the correlations for the summer data is steeper at the shortest separation distances and not as steep at the longest separations.

\section{Estimation of observational error}

Extrapolation to zero separation of the structure values computed in this study yields an estimate of the random measurement error for the $u$ and $v$ components of INS-AIDS winds. Although isotropic and homogeneous conditions are necessary for this technique to be appropriate, the discussion in the previous section has shown that these conditions are valid when the separation interval is not large (approximately $200 \mathrm{~km}$ or less). Therefore, in the extrapolation procedure employed here, only structure values from distance intervals through $225 \mathrm{~km}$ have been used. The function chosen for extrapolation purposes was the cosine function translated to the proper quadrant and modified to fit the scale of the farthest distance interval. The resulting curves and intercepts for each month and component can be seen in Fig. 11. The curves were fitted to the data using the least-squares method. The intercept is equal to twice the mean squared error associated with the instrument (see Eq. [5]). Table 2 presents the corresponding values of the error standard deviation calculated from each intercept. This estimate of the measurement error is noticeably different between the two seasons but within each season, the $u$ and $v$ component values are quite similar. The vector-error values computed using the components from the August data are $1 \mathrm{~m} \cdot \mathrm{s}^{-1}$ larger than that computed from the January data. This difference reflects the more variable nature of summer-season winds at smaller 

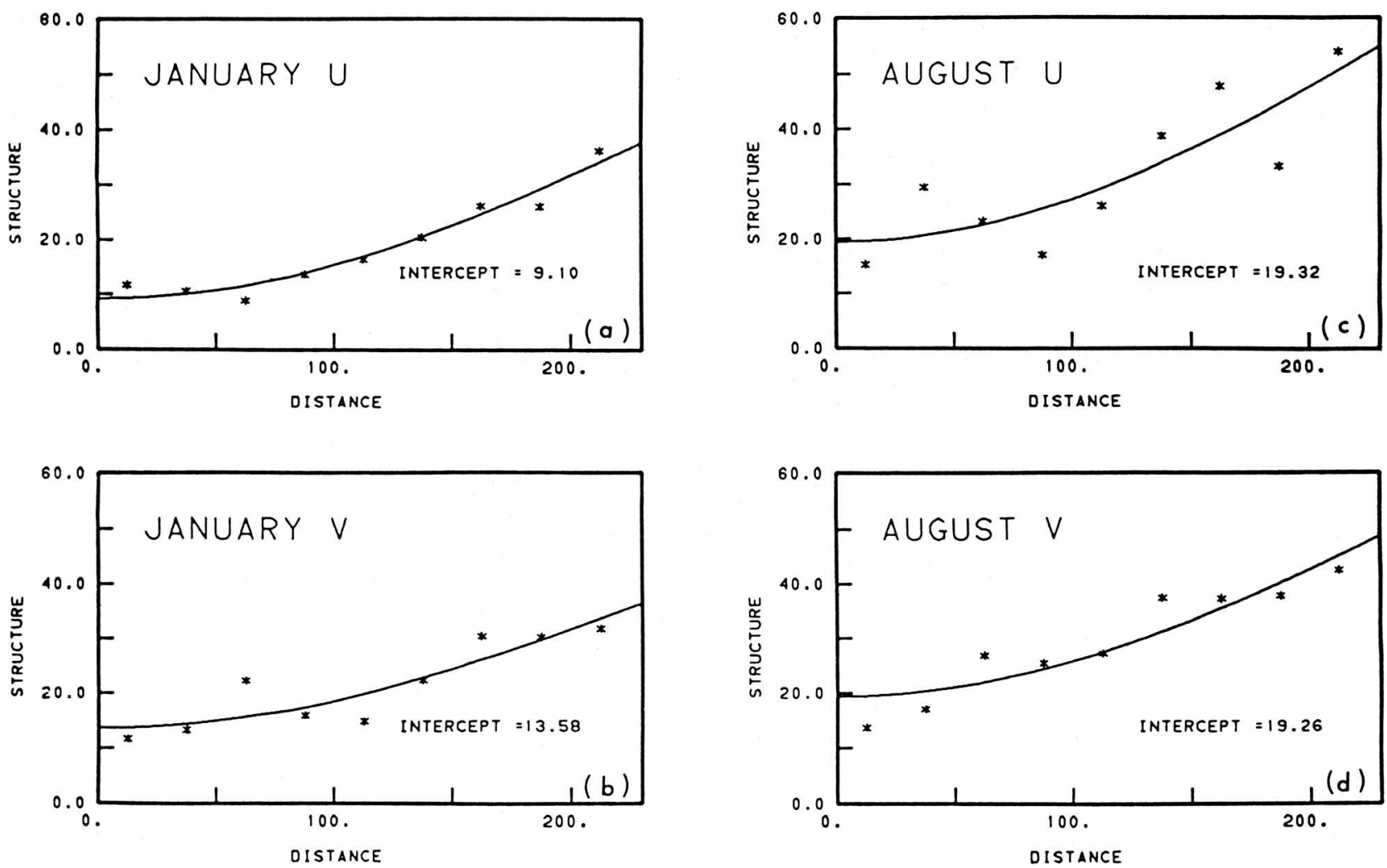

FIG. 11. Curves fit to structure values through $225 \mathrm{~km}$ for (a) January $u$ component, (b) January $v$ component, (c) August $u$ component, and (d) August $v$ component.

scales. The inaccuracies inherent in the extrapolation procedure as well as the degree to which the assumptions of homogeneity and isotropy are not observed are illustrated by comparing the values of the component wind errors from Table $2\left(2.1-3.1 \mathrm{~m} \cdot \mathrm{s}^{-1}\right)$ with component error results from two studies of INS-based aircraft wind measurements. Koscielny and Duchon (1975) used data from the INS on board the NCAR Electra to derive a component error of $1.4 \mathrm{~m} \cdot \mathrm{s}^{-1}$. Lenschow (1972), using information provided by the NCAR Buffalo aircraft, estimated that individual components of the wind could be measured within $1.0 \mathrm{~m} \cdot \mathrm{s}^{-1}$ accuracy.

The INS-AIDS results can also be compared to estimates derived by Fuelberg and Meyer (1984). As part of their study of AVE-SESAME I mesoscale radiosonde data, they used the method of extrapolating structure functions to zero separation to estimate the random measurement error in radiosonde wind-speed observations. Their results for 300 and $200 \mathrm{mb}$ are also presented in Table 2. Note that errors in wind speed do not take errors in direction into account as vector errors do. The higher values of measurement error estimated for the radiosonde wind speed provide evidence for the belief that INS-derived winds are more accurate at this level than radiosondes. The winds collected by Fuelberg and Meyer (1984) were not restricted by wind speed as the aircraft winds used in this study were. Figure 8 demonstrates that the effect of calculating structure functions using reports with higher windspeed values is to increase the structure values. Pairs of reports with high wind speeds are more likely to be in the vicinity of the strong horizontal gradients of a jet stream and thus in a region where the assumptions of homogeneity and isotropy are not valid. These facts cloud the comparison presented in Table 2 as all or part of the difference may be due to this cause.

The measurement error associated with an observing system is an estimate of the uncertainty of that instrument only in measuring a quantity at a particular point in space and time. The observational error that must be specified for every instrument and data type used by the optimum-interpolation analysis performed at the National Meteorological Center must account not only for this type of error but also for the error of unrepresentativeness; that is, the inability of the instrument to produce a value characteristic of the length and time scales unresolvable by the analysis. Structure functions can be used to determine the magnitude of this type of error.

The value of a structure function at a given distance or distance interval represents, by definition (Eq. [3]), the variability of the measured field for all length scales less than that given distance. This variability includes both the measurement error and the error of unrepresentativeness. Therefore, if the resolvable scale of a particular analysis procedure is known, the structure value at the distance representing the resolvable scale is equal to twice the observational-error variance for the particular instrument used to construct the structure function. For example, if the resolvable scale of an analysis has been determined to be $300 \mathrm{~km}$, then the value of the structure function at $300 \mathrm{~km}$ for both the $u$ and $v$ components can be obtained from either Fig. 2 or 3. From Fig. 2, the 


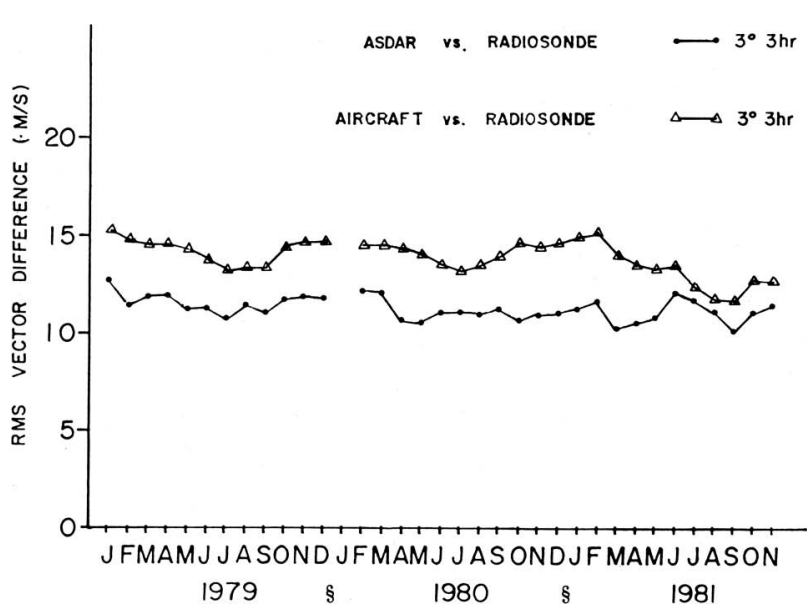

FIG. 12. Monthly rms vector wind colocation differences between radiosondes and ASDAR-measured winds for a three-degreethree-hour window (solid line) and between radiosondes and aircraft-measured winds for a three-degree-three-hour window (triangles) for the time period January 1979-November 1981.

January data, the structure values for both components at $300 \mathrm{~km}$ are about the same: approximately $56 \mathrm{~m}^{2} \cdot \mathrm{s}^{-2}$. The observational-error standard deviation for each component is computed as $(56 / 2)^{1 / 2}$, or $5.3 \mathrm{~m} \cdot \mathrm{s}^{-1}$. The observational vector error standard deviation computed from the two component errors is $7.5 \mathrm{~m} \cdot \mathrm{s}^{-1}$. For the August data, at $300 \mathrm{~km}$, the values are about the same (Fig. 3).

This knowledge of the observational error of INS-AIDS winds can be used to estimate the observational error of radiosonde winds when combined with the results of colocation studies. Monthly colocation statistics computed between ASDAR and radiosonde winds are presented in Fig. 12 (C. Vlcek, personal communication) for 1979-81. After November 1981, the number of available colocated radiosonde and ASDAR wind measurements dropped off significantly. The root-mean-square vector wind colocation differences above $500 \mathrm{mb}$ within a three-degree and three-hour window are plotted with a solid line. In practice, most of the reports in the above-500-mb group are from the cruising altitude of the aircraft, which is about 10000 meters for widebodied jets. The values range from 10 to $12 \mathrm{~m} \cdot \mathrm{s}^{-1}$. Since these colocation differences are between two different types of instruments, the observational error from both is represented in the value. However, since the observational vector error of the ASDAR winds can be obtained from Fig. 2 or 3 for a three-degree resolution, it is possible to separate it from the total error represented by the colocation difference and assign an observational error to radiosonde winds for this level. The following simple formula is used:

$$
\sigma_{\mathrm{COL}}{ }^{2}=\sigma_{R}^{2}+\sigma_{A}{ }^{2} .
$$

$\sigma_{\mathrm{COL}}$ represents the vector colocation error, $\sigma_{R}$ is the radiosonde observational vector error and $\sigma_{A}$ is the ASDAR observational vector error. This formula assumes that the radiosonde and ASDAR errors are independent. Using a representative colocation error of $11 \mathrm{~m} \cdot \mathrm{s}^{-1}$ and the INS-AIDS observational vector error at $300 \mathrm{~km}$ computed in the previous paragraph, Eq. (9) produces an observational vector error for radiosonde winds of $8.0 \mathrm{~m} \cdot \mathrm{s}^{-1}$. This value is only valid for a three-degree resolution at the flight level.

The triangles in Fig. 12 represent vector wind differences from colocations between radiosondes and conventional aircraft wind measurements for the same time period. The separation window is three degrees and three hours. These values imply superiority of ASDAR over conventional aircraft wind reports as they are at least $2 \mathrm{~m} \cdot \mathrm{s}^{-1}$ higher than the comparable ASDAR colocations (solid line) throughout most of the period. An estimate of the observational vector error of these aircraft winds can be determined by removing the radiosonde vector wind error just computed from a value of the colocation difference representative of the period, about 13 $\mathrm{m} \cdot \mathrm{s}^{-1}$, using an equation similar to Eq. (9). The value obtained in this way is $10 \mathrm{~m} \cdot \mathrm{s}^{-1}$. It is important to remember that this value as well as the values computed for the ASDAR and radiosonde vector winds are all estimates of the observational error of the instrument, not the measurement error, and as such are representative of a particular resolution, in this case three degrees.

Previous studies done at the National Meteorological Center have used other methods to assign a representative observational error value to INS-AIDS winds. McInturff (1977) compared AIDS winds with geostrophic winds computed from analyses for two flight routes and obtained vector wind differences of 13 and $9 \mathrm{~m} \cdot \mathrm{s}^{-1}$. McPherson and Rasch (1977) did not make statistical comparisons but reported that "direction and speed are in good agreement" compared to radiosondes. A comparison of 20 days of ACARS data with radiosonde data by $\mathrm{Yu}$ (personal communication) revealed root-mean-square vector wind colocation differences using a three-degree-three-hour window to have an average value of about $11 \mathrm{~m} \cdot \mathrm{s}^{-1}$. This result is very similar to the ASDAR colocations in Fig. 12.

\section{Summary and conclusion}

In this study structure functions of $u$ and $v$ wind components were constructed from paired INS-AIDS wind observations at the aircrafts' flight levels. Reports from two months representing winter and summer seasons were analyzed separately. Extrapolation of these structure functions to zero separation produced an estimate of the random measurement error for each component. The component errors ranged from 2.1 to $3.1 \mathrm{~m} \cdot \mathrm{s}^{-1}$. The vector errors computed from the component errors were $3.4 \mathrm{~m} \cdot \mathrm{s}^{-1}$ using the January data and $4.4 \mathrm{~m} \cdot \mathrm{s}^{-1}$ using the August data.

The principal objective of this study was to provide a means of estimating the observational error of this type of wind report. Observational error, including both measurement error and the error of unrepresentativeness, must be specified for every type of observation used by an OI analysis procedure. The latter type of error is a function of the scale for which the observation must be representative. Different analysis procedures providing initial conditions for different numerical models may have different requirements for resolvable scale. Structure functions provide an estimate of the variability in a field measured by a particular instrument as a function of distance. This variability estimate represents the observational error because it includes both the measure- 
ment error and the error of unrepresentativeness. All that is needed is knowledge of the appropriate resolvable scale of the analysis to be able to determine the proper observational error for an instrument from a plot of the structure of a variable as measured by that instrument. This method is simple and informative and resulted in values that were shown to be quite reasonable. One must keep in mind, however, the limitations introduced by the homogeneity and isotropy assumptions. For a 300-km resolution, the component error of INS-AIDS winds for both seasons was estimated to be 5.3 $\mathrm{m} \cdot \mathrm{s}^{-1}$.

The availability of colocation statistics differencing radiosondes with INS-ASDAR winds allowed the observational error of the radiosonde winds to be computed. These results as well as measurement-error results from another structure function study (Fuelberg and Meyer, 1984) demonstrated that INS-AIDS/ASDAR winds are very competitive with radiosondes. They are a valuable addition to the observational data base.

Acknowledgments. The author wishes to thank Dr. Paul Julian for his review of this work and the resulting helpful criticisms. The patience and encouragement of Dr. Ronald McPherson are also gratefully acknowledged. Thanks go to C. Burley for drafting the figures.

The study was partly funded by the NOAA office of Climate and Atmospheric Research.

\section{References}

Barnes, S. L., and D. K. Lilly, 1975: Covariance analysis of severe storm environments. Preprints, Ninth Conf. Severe Local Storms, Norman, Ok., American Meteorological Society, 301-306.
Bergman, K., 1979: Multivariate analysis of temperatures and winds using optimum interpolation. Mon. Wea. Rev., 107, 1432-1444.

Fuelberg, H. E., and P. J. Meyer, 1984: An analysis of the AVESESAME I period using statistical structure and correlation functions. Mon. Wea. Rev., 112, 1562-1576.

Gandin, L. S., 1963: Objective analysis of meteorological fields. Translated from Russian, Israel Program for Scientific Translations, Jerusalem, 242 pp. [NTIS TT-65-50007.]

Hillger, D. W., and T. H. Vonder Haar, 1979: An analysis of satellite infrared soundings at the mesoscale using statistical structure and correlation functions. J. Atmos. Sci., 36, 287-305.

Julian, P. R., 1983: On the use of observational error data in FGGE analysis. ECMWF Tech. Memo. 76, 24 pp.

- , and R. Steinberg, 1975: Commercial aircraft as a source of automated meteorological data for GATE and DST. Bull. Amer. Meteor. Soc., 56, 243-251.

Koscielny, A. J., and C. E. Duchon, 1975: Estimation of mean square reconstruction error of the Schuler error in an inertial navigation system. Preprints, Fourth Conf. Probability and Statistics in Atmospheric Science, Tallahassee, American Meteorological Society, Boston, Mass., 108-113.

Lenschow, D. H., 1972: The measurement of air velocity and temperature using the NCAR Buffalo aircraft measuring system. NCAR-TN-EDD-74, 39 pp.

Lorenc, A. C., 1984: Analysis methods for the quality control of observations. Met 011 Tech. Note No. 194, 34 pp.

McInturff, R. M., 1977: A comparison of AIDS data from the Concorde with data obtained from rawinsonde and satellite. Office Note 164, Part I, NMC, Washington, D.C., 8 pp.

McPherson, R. D., and G. E. Rasch, 1977: An example of ASDAR winds in the NMC analysis. Office Note 164, Part II, NMC, Washington, D.C., 6 pp.

Maddox, R. A., and T. H. Vonder Haar, 1979: Covariance analysis of satellite-derived mesoscale wind fields. J. Appl. Meteor., 18, 1327-1334. announcements (continued from page 150)

mitted any time. Postdoctoral appointments will be made from applications submitted by 30 March 1986. Applications should include four professional references. Send the required information to Gene Martin, Corporate Projects, UCAR, P.O. Box 3000, Boulder, CO 80307; telephone (303) 497-8999.

\section{meetings of interest}

31 March-4 April 1986. The Optical Society of America is sponsoring the OSA Spring '86 Conference from 31 March to 4 April 1986. Four meetings are planned. A meeting entitled "Holography" will be held from 31 March to 2 April. The second meeting, entitled "Quantum Limited Imaging and Image Processing," will also be held from 31 March to 2 April. "Signal Recovery and Synthesis II" will run from 2 to 4 April, and "Meteorological Optics" will be held on 3-4 April. For more information contact the Optical Society of America, Spring Conference, 1816 Jefferson Place, N.W., Washington, D.C. 20036.

8-14 June 1986. The American Solar Energy Society is sponsoring the 11th National Passive Solar Conference, to be held in conjunction with the Society's Annual Meeting, on 8-14 June 1986 at the University of Colorado in Boulder. Technical sessions of the conference will be presented on 9, 10, and 11 June 1986. Session topics will include solar radiation, agriculture, architecture, engineering, bio- and chemical conversion, physics, wind, and power utility use. For more information contact the American Solar Energy Society, Inc., The
U.S. Section of the International Solar Energy Society, 2030 17 th St., Boulder, CO 80302.

June 1986. The Israel Society for Ecology and Environmental Quality Sciences is sponsoring the Third International Conference on Ecology and Environmental Quality in June 1986 in Jerusalem, Israel: For further information contact the Israel Society for Ecology and Environmental Sciences, The Hebrew University of Jerusalem, P. O. Box 1172, Jerusalem, Israel.

7-12 September 1986. The International Glaciological Society is sponsoring the IGS Symposium on Remote Sensing Glaciology on 7-12 September 1986 in Cambridge, England. For further details contact H. Richardson, International Glaciological Society, Lensfield Rd., Cambridge CB2 lER, England.

7-12 September 1986. The Second International Conference on Paleoceanography is scheduled to take place on 7-12 September 1986 in Woods Hole, Massachusetts. Contact W. A. Berggren, Department of Geology and Geophysics, Woods Hole Oceanographic Institution, Woods Hole, MA 02543 for more information.

September 1987. The International Congress on the History of Oceanography (ICHO IV) will be held in September 1987 in Hamburg, the Federal Republic of Germany. For more information contact the Deutsche Gesellschaft fr Meeresforschung, ICHO IV, Bundesstrasse 55, D-2000, Hamburg 13, FRG. 